\title{
Perda auditiva leve: desempenho no Teste da Habilidade de Atenção Auditiva Sustentada*****
}

\author{
Mild hearing loss: performance in the Sustained Auditory Attention \\ Ability Test
}

\author{
Maria Fernanda Capoani Garcia Mondelli* \\ Fernanda Ribeiro Pinto de Carvalho** \\ Mariza Ribeiro Feniman*** \\ José Roberto Pereira Lauris****
}

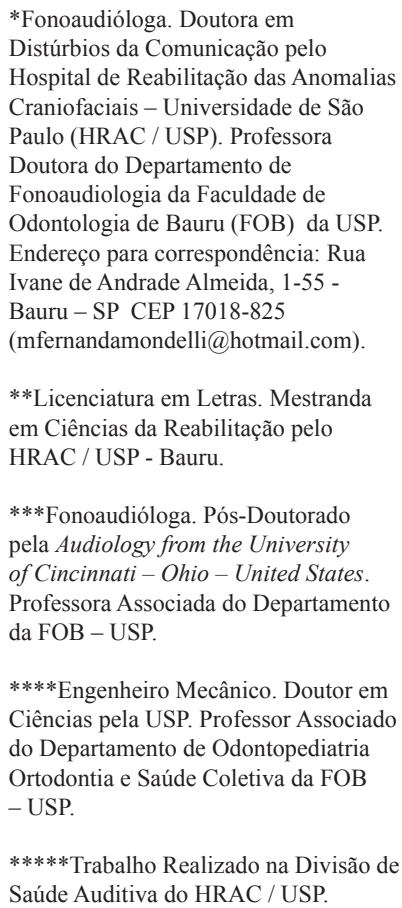

*Fonoaudióloga. Doutora em Distúrbios da Comunicação pelo Hospital de Reabilitação das Anomalias Craniofaciais - Universidade de São Paulo (HRAC / USP). Professora

Doutora do Departamento de Fonoaudiologia da Faculdade de Odontologia de Bauru (FOB) da USP. Endereço para correspondência: Rua Ivane de Andrade Almeida, 1-55 Bauru - SP CEP 17018-825 (mfernandamondelli@hotmail.com).

**Licenciatura em Letras. Mestranda em Ciências da Reabilitação pelo HRAC / USP - Bauru.

***Fonoaudióloga. Pós-Doutorado pela Audiology from the University of Cincinnati - Ohio - United States. Professora Associada do Departamento da FOB - USP

****Engenheiro Mecânico. Doutor em Ciências pela USP. Professor Associado do Departamento de Odontopediatria Ortodontia e Saúde Coletiva da FOB - USP.

*****Trabalho Realizado na Divisão de Saúde Auditiva do HRAC / USP.

Artigo Original de Pesquisa

Artigo Submetido a Avaliação por Pares

Conflito de Interesse: não

\begin{abstract}
Background: hearing loss during childhood is considered a risk factor for developmental delay. Aim: to verify the performance of children diagnosed with mild hearing loss, conductive and sensorineural, in the Sustained Auditory Attention Ability Test (SAAAT). The purpose of the study was to verify if the test is influenced by the presence of hearing impairment. Method: a clinical study of the SAAAT considering three groups: Group 1 (G1) control group composed by children with normal hearing, Group 2 (G2) composed by children with mild bilateral sensorineural hearing loss and Group $3(\mathrm{G} 3)$, composed by children with mild bilateral conductive hearing loss. Participants were children with ages between 7 and 11 years; 30 children in each group. A prospective study. Interventions: Pure Tone Audiometry, Acoustic Imittance Measures and SAAAT. Result: children with sensorineural and conductive hearing loss presented a lower performance in all of the SAAT results when compared to the control group. Conclusion: the SAAAT was influenced by the presence of mild hearing impairment, considering that the greatest influence was observed in the presence of sensorineural hearing loss.
\end{abstract}

Key Words: Attention; Hearing Loss; Sensorineural; Hearing Loss; Conductive.

\section{Resumo}

Tema: a perda auditiva na infância é um fator de risco para o atraso no desenvolvimento. Objetivo: verificar o desempenho de crianças diagnosticadas com perda auditiva de grau leve - condutiva e sensorioneural, no Teste da Habilidade de Atenção Auditiva Sustentada (THAAS), visando constatar se este teste sofre influência da presença de uma perda auditiva. Método: estudo clínico do THAAS em 3 grupos: Grupo 1 (G1) grupo controle formado por crianças com audição normal, Grupo 2 (G2) crianças com deficiência auditiva sensorioneural bilateral de grau leve e Grupo 3 (G3) composto por crianças com perda auditiva condutiva bilateral de grau leve. Estudo prospectivo. Participantes: 90 crianças com idade entre 7 e 11 anos de idade, sendo 30 de cada grupo. Intervenções: Audiometria Tonal Limiar, Imitanciometria e THAAS. Resultados: os grupos sensorioneural e condutivo apresentaram desempenho inferior ao grupo controle em todas as respostas do THAAS. Conclusão: o THAAS sofreu influência das perdas auditivas de grau leve, condutivas e sensorioneurais, sendo o pior comprometimento para perdas sensorioneurais.

Palavras-Chave: Atenção; Perda Auditiva Neurossensorial; Perda Auditiva Condutiva. 


\section{Introdução}

A perda auditiva na infância é um importante problema de saúde pública, sendo estimado que uma criança a cada 1000 ou 6000 nasce com deficiência auditiva. A perda auditiva de grau leve é mais significativa na faixa escolar, sendo o tipo condutivo o mais observado ${ }^{1}$. Perda auditiva condutiva de grau leve a moderado e inabilidades funcionais a curto-prazo, especialmente em ambiente ruidoso ${ }^{2}$, são frequentes em crianças com Otite Média com Efusão (OME).

Trabalhos $^{3-4}$ têm demonstrado significante associação entre a OME e a habilidade de atenção da criança. Um estudo realizado ${ }^{5}$ com crianças com e sem múltiplos episódios de otite média bilateral recorrente demonstrou prejuízo na habilidade de atenção, quando as crianças com histórico de OME foram submetidas à escuta dicótica. A OME interfere na habilidade de persistir e focar a atenção em eventos auditivos ${ }^{6}$. No entanto, controvérsias permanecem sobre os efeitos da OME no desenvolvimento da criança a longo prazo $^{7-8}$. As implicações da otite média e perda auditiva condutiva são menos claras que àquelas associadas com perda auditiva sensório-neural ${ }^{9}$. Assim, significativos déficits de linguagem e acadêmicos são relatados em pesquisas ${ }^{10-11} \mathrm{com}$ perda auditiva sensório-neural bilateral leve, contrapondo o observado em estudo ${ }^{12}$ que não encontrou forte evidência.

A atenção é um processo multimodal ${ }^{13}$, sendo a habilidade de atenção auditiva de fundamental importância para o processamento da informação selecionada e para a aprendizagem de novas tarefas. Entre os tipos de atenção auditiva, é a atenção sustentada que faz com que o sujeito persista, mantenha e complete uma tarefa em um dado período de tempo ${ }^{14}$.

Considerando o papel fundamental da audição no desenvolvimento da fala, linguagem, aprendizagem e habilidades auditivas, julgou-se necessário realizar um estudo prospectivo do efeito da presença de uma perda auditiva na atenção auditiva de crianças.

O presente estudo tem como objetivo verificar o desempenho de crianças diagnosticadas com perda auditiva leve no teste de habilidade de atenção auditiva sustentada (THAAS).

\section{Método}

A presente pesquisa foi realizada na Divisão de Saúde Auditiva de um hospital especializado em deficiência auditiva, no período de fevereiro de 2006 a março de 2007. Todos os procedimentos e termos de consentimento foram previamente aprovados pelo Comitê de Ética em Pesquisa, protocolo número 101/2006.

Neste trabalho a amostra constituiu-se de 60 crianças de ambos os gêneros, com deficiência auditiva de grau leve, que preenchiam os seguintes critérios de inclusão e exclusão.

Os critérios de inclusão foram: As crianças estavam entre as idades de 7 a 11 anos e possuíam diagnóstico de perda auditiva sensório-neural e condutiva de grau leve.

Os critérios de exclusão foram: não apresentar síndromes, anomalias craniofaciais, possuírem características de transtorno e déficit de atenção e hiperatividade, apresentarem infecção das vias aéreas superiores em situação de exame e dificuldade para compreender os testes auditivos propostos.

O grupo de estudo foi subdividido em 2 subgrupos, pacientes que apresentavam perda auditiva sensório-neural (SN) e, que apresentavam perda auditiva condutiva (Cond). $\mathrm{O}$ grupo controle (Contr) foi constituído de crianças voluntárias da mesma faixa etária, que não apresentavam deficiência auditiva, isto é, não apresentavam limiares tonais aéreos rebaixados (acima de 15 dBNA) na audiometria ${ }^{15}$.

A Tabela 1 apresenta a distribuição das crianças amostradas, segundo a idade, o gênero e o grupo.

TABELA 1. Distribuição de frequência das crianças segundo o grupo, a idade e o gênero.

\begin{tabular}{c|c|c|c|c|c|c}
\hline & \multicolumn{5}{c}{ Grupos } \\
\hline \multirow{2}{*}{$\begin{array}{c}\text { Idade } \\
\text { (Anos) }\end{array}$} & \multicolumn{2}{|c|}{ Controle } & \multicolumn{2}{c}{ Sensório Neural } & \multicolumn{2}{c}{ Condutivo } \\
\cline { 2 - 7 } & Feminino & Masculino & Feminino & Masculino & Feminino & Masculino \\
\hline 7 & 2 & 4 & 5 & 2 & 3 & 1 \\
8 & 6 & 3 & 4 & 2 & 5 & 2 \\
9 & 3 & 3 & 3 & 3 & 2 & 5 \\
10 & 4 & 4 & 4 & 6 & 5 & 7 \\
11 & 0 & 1 & 0 & 1 & 0 & 0 \\
TOTAL & 15 & 15 & 16 & 14 & 15 & 15 \\
\hline
\end{tabular}


Após preencherem os critérios de inclusão e exclusão, as crianças foram submetidas a uma entrevista dirigida, em forma de questionário, visando verificar a sua saúde auditiva, status da audição, bem como os aspectos relacionados à habilidade de atenção e impulsividade da criança. $\mathrm{O}$ exame otorrinolaringológico deu ênfase à otoscopia. Todas as crianças foram submetidas à Audiometria Tonal Liminar, à Logoaudiometria e Imitanciometria, por meio do audiômetro, modelo AD28 e fone TDH - 39 - padrão ANSI, 1969 e imitanciômetro modelo AZ 7, respectivamente, e ao THAAS com objetivo de avaliar a atenção auditiva sustentada da criança.

O THAAS $^{16}$ é um teste utilizado para avaliar a atenção auditiva sustentada, no qual a criança foi instruída verbalmente, que deveria responder levantando a mão para apenas um estímulo auditivo específico (palavra alvo), mantendo a atenção e concentração na tarefa por um período de tempo prolongado. Consiste na apresentação, por meio de fones de orelha, de uma lista de 100 palavras monossilábicas (contendo as 20 ocorrências da palavra alvo "não"). Esta lista (gravada em CD) foi apresentada seis vezes sem interrupção. As respostas foram registradas em protocolo específico, em frente a cada palavra do teste para qual a criança levantou a mão. Este teste foi realizado em cabina acústica, com auxílio de um CD player (Compact Player D-171, Sony) acoplado a um audiômetro de dois canais (Midimate 622-Madsen Eletronics), a uma intensidade de 50 dBNS (deciBel Nível de Sensação), considerando a média dos limiares auditivos de 500, 1000 e 2000 $\mathrm{Hz}$, para cada orelha, de maneira binaural e diótica.

No desempenho do THAAS foram considerados a pontuação total dos erros e o decréscimo de vigilância.
Definiu-se pontuação total a contagem do número de erros de desatenção acrescida do número de erros de impulsividade, sendo considerado erro de desatenção, quando a criança não levantou a mão em resposta à palavra "não" antes da apresentação da palavra seguinte; e erro de impulsividade quando a criança levantava a mão para outra palavra ao invés da palavra "não".

Definiu-se como decréscimo de vigilância o declínio na atenção, ou seja, a diferença entre o número de respostas corretas para a palavra "não" na $1^{\mathrm{a}}$ apresentação e o número de respostas corretas obtidas na $6^{\mathrm{a}}$ apresentação.

Para comparar os grupos foi utilizado inicialmente um modelo de regressão linear com os fatores: idade (anos), gênero e grupo, para cada variável estudada ${ }^{17}$. Como os fatores gênero e idade não foram estatisticamente significantes $(\mathrm{p}$ $>0,05)$, optou-se por refazer a análise por meio de um modelo de análise de variância com apenas o fator grupo ${ }^{17}$. Para realizar as comparações post$h o c^{17}$ foi utilizado a correção de Tukey.

\section{Resultados}

Com base nos resultados obtidos no THAAS elaborou-se a Tabela 2 demonstrando a análise descritiva dos erros de impulsividade, de desatenção, da pontuação total e do decréscimo de vigilância, respectivamente, dos três grupos, e resultado da Análise de Variância (ANOVA) e teste de Tukey para a comparação entre eles.

Para os erros de impulsividade, de desatenção e para a pontuação total não foi encontrada diferença estatística significativa entre os grupos Contr e o Cond $(p=0,069),(p=0,504),(p=0,114)$ respectivamente, porém foi observada entre os Contr e SN $(p<0,001)$ e o SN e Cond ( $<<0,001)$.

Para o decréscimo de vigilância não foi encontrada diferença estatística significativa entre os grupos SN e o Cond ( $\mathrm{p}=0,603)$, porém foi observado para os grupos Contr e SN $(\mathrm{p}<0,001)$ e o Contr e Cond $(\mathrm{p}<0,001)$. 
TABELA 2. Descrição da impulsividade, desatenção, pontuação total e decréscimo de vigilância dos três grupos, e resultado da Análise de Variância (ANOVA) e teste de Tukey para a comparação entre eles.

\begin{tabular}{|c|c|c|c|c|c|c|c|}
\hline Grupo & Média & $\mathrm{Dp}$ & Mínimo & Mediana & Máximo & ANOVA & Tukey \\
\hline impulsividade Contr & 3 & 2,28 & 0 & 2 & 10 & \multirow{4}{*}{$\mathrm{p}<0,001 *$} & Contr x SN: $\mathrm{p}<0,001^{*}$ \\
\hline impulsividade SN & 10 & 6,6 & 0 & 9,5 & 28 & & Contr $\mathrm{x}$ Cond: $\mathrm{p}=0,069 \mathrm{~ns}$ \\
\hline impulsividade Cond. & 5,6 & 3,15 & 0 & 5,5 & 11 & & SN $x$ Cond: $p=0,001^{*}$ \\
\hline desatenção Contr & 4,7 & 4,9 & 0 & 2 & 19 & & Contr $\mathrm{x}$ SN: $\mathrm{p}<0,001^{*}$ \\
\hline desatenção SN & 13,7 & 9,38 & 0 & 13 & 36 & \multirow{4}{*}{$\mathrm{p}<0,001^{*}$} & Contr $\mathrm{x}$ Cond: $\mathrm{p}=0,504 \mathrm{~ns}$ \\
\hline desatenção Cond & 6,6 & 4,17 & 0 & 7 & 19 & & SNxCond: $\mathrm{p}<0,001^{*}$ \\
\hline pontuação Total Contr & 7,7 & 5,69 & 0 & 5,5 & 23 & & Contr $x$ SN: $\mathrm{p}<0,001^{*}$ \\
\hline pontuação Total SN & 23,7 & 12,41 & 3 & 21,5 & 61 & & Contr $\mathrm{x}$ Cond: $\mathrm{p}=0,114 \mathrm{~ns}$ \\
\hline pontuação Total Cond & 12,2 & 5,85 & 4 & 12 & 28 & \multirow{4}{*}{$\mathrm{p}<0,001 *$} & SN x Cond: $\mathrm{p}<0,001^{*}$ \\
\hline decréscimo de Vigilância Contr & 0,5 & 0,73 & 0 & 0 & 2 & & Contr x SN: $\mathrm{p}<0,001^{*}$ \\
\hline decréscimo de Vigilância SN & 3,3 & 2,76 & 0 & 3 & 11 & & Contr $\mathrm{x}$ Cond: $\mathrm{p}<0,001^{*}$ \\
\hline decréscimo de Vigilância Cond & 3,9 & 2,73 & 0 & 3,5 & 10 & & SN x Cond: $\mathrm{p}=0,603 \mathrm{~ns}$ \\
\hline
\end{tabular}

* = diferença estatisticamente significante $(\mathrm{p}<0,05) ; \mathrm{ns}=$ diferença estatisticamente não significante; $\mathrm{SN}=$ sensório-neural; Contr = controle; Cond = condutiva.

\section{Discussão}

Com relação à idade e ao gênero (Tabela 1) o grupo Contr e os grupos com deficiência auditiva $\mathrm{SN}$ e Cond foram equiparados da maneira mais próxima possível para que houvesse maior fidelidade na comparação dos resultados. Uma vez que o controle do impulso desenvolve-se mais cedo no gênero feminino ${ }^{18}$, poderia se esperar, neste estudo, escores maiores (piores) de impulsividade para os meninos, porém não houve diferença significativa entre os gêneros, em concordância a outros trabalhos ${ }^{16,19-20}$. Contrariamente, um estudo $^{21}$ aponta escores maiores de impulsividade para o gênero masculino. Assim, a ausência de significância encontrada no presente trabalho para todos os tipos de erros no THAAS (impulsividade, desatenção, pontuação total e decréscimo de vigilância), no que se refere ao gênero, foi concordante com trabalhos anteriormente realizados ${ }^{16,20}$.

No que se refere aos grupos amostrados, com ou sem perda de audição, maior impulsividade foi observada no grupo SN, seguida pelo grupo Cond, quando comparado ao grupo Contr (Tabela 2). Assim, dados deste estudo leva a se pensar que as crianças com perda sensório-neural e com perda auditiva condutiva são mais impulsivas que aquelas com audição dentro dos padrões de normalidade (grupo Contr). Nenhuma informação relacionando a impulsividade e tipo de perda auditiva foi encontrado na literatura consultada.
Semelhante ao observado para o erro de impulsividade, escores maiores (piores) com diferença estatisticamente significativa foi obtida para o erro de desatenção para as crianças com deficiência auditiva sensório-neural e para as com perda auditiva condutiva, quando comparados aos obtidos às do grupo Contr. A desatenção é exibida pelas crianças que apresentam dificuldade de decodificação léxica, que freqüentemente demoram no processamento do estímulo e perdem a palavra completamente ou exibem uma resposta demorada ${ }^{10}$. É um problema que faz com que a criança perca ou não registre informações em sua memória para processá-las ${ }^{22}$. Assim, elas gastam mais tempo nas tarefas escolares, por exemplo, tentando recuperar qual informação perderam (devido a sua desatenção), e como resultado o processamento da informação é atrasado. A literatura tem relatado que as crianças com deficiência auditiva condutiva apresentam longos períodos de privação sensorial em decorrência de alterações da orelha média ${ }^{23-24}$ podendo interferir na habilidade da atenção ${ }^{3-4,25}$, influenciar negativamente as estruturas e função do sistema auditivo central, acarretar efeitos adversos que comprometem o desenvolvimento cognitivo, lingüístico e ocasionar perda auditiva permanente ${ }^{26}$. No entanto, efeitos da OME a longo prazo permanecem inconclusivos ${ }^{2}$. Ressalta-se que, um estudo ${ }^{27}$ prospectivo de 10 anos, contraria a hipótese de prejuízo a longo prazo no desenvolvimento da linguagem. 
Semelhante ao observado com o grupo de crianças sem perda de audição (grupo Contr), neste estudo, os grupos com deficiência auditiva (grupo $\mathrm{SN}$ e grupo Cond) foram mais desatentos que impulsivos. Este achado é consoante com achados na literatura ${ }^{20,28}$. A importância desse resultado recai no processo terapêutico, tendo em vista que a recomendação de estratégias compensatórias específicas está na dependência do tipo de erro apresentado pela criança. No entanto, autores ${ }^{29}$ sugerem os erros de impulsividade como fator chave para elaborar um diagnóstico diferencial de transtorno de processamento auditivo ou transtorno de processamento auditivo e transtorno de déficit de atenção e hiperatividade.

Tendo em vista que a pontuação total no THAAS é quantificada considerando o número de erros de impulsividade e de desatenção, nota-se que seu valor e estudo estatístico encontram-se análogos aos escores de desatenção e de impulsividade, demonstrando que as crianças com perda auditiva sensório-neural (grupo $\mathrm{SN}$ ) e condutiva (grupo Cond) apresentaram desempenho pior que as sem qualquer alteração auditiva (grupo Contr). No entanto, diferença estatisticamente significativa entre os grupos (Cond e SN) foi observada, tendo em vista que o grupo SN apresentou um escore médio $(23,7)$ três vezes o número de erros, se comparado ao grupo Contr e, praticamente, o dobro do obtido pelo grupo Cond $(12,2)$. Assim, é possível sugerir que a deficiência auditiva sensórioneural propicie maior comprometimento da atenção auditiva sustentada, em relação à deficiência auditiva condutiva, que apesar da inconsistência na recepção sonora, apresentou melhores escores nas respostas ${ }^{30}$.

\section{Referências Bibliográficas}

1. Souza Neto OM. Diagnóstico precoce na deficiência auditiva.
ACTA OTL/Técnicas em Otorrinolaringologia. 2007;25(1):2-3.

2. Williamson I, Little P. Otitis media with effusion: the long and winding road? Arch Dis Child. 2008;93(4):268-9.

3. Feagans L, Sanyal M, Henderson F, Collier A, Appelbaum M. Relationship of middle eat disease in early childhood to later narrative and attention skills. J Pediatr Psychol. 1987;12(4):581-94.

4. Feagans LV, Kipp E, Blood I. The effects of otitis media on the attention skills of day-care-attending toddlers. Dev Pshychol. 1994;30:701-8.
Decréscimo de vigilância é o declínio na atenção que ocorre durante o tempo em uma tarefa de vigilância. Sendo o THAAS um teste de vigilância auditiva, os resultados do presente estudo demonstram que as crianças com deficiência auditiva, seja sensório-neural ou condutiva apresentaram escores estatisticamente significativos piores $(3,3$ e 3,9 , respectivamente) que as sem perda de audição $(0,5)$. Este resultado vem caracterizar um déficit na atenção auditiva sustentada se comparado ao valor médio obtido em estudos anteriormente realizados com crianças ouvintes normais ${ }^{16,20,28}$, que encontraram menor declínio na atenção de crianças sem déficit de atenção e hiperatividade, quando comparado ao das crianças com este déficit, durante uma tarefa de vigilância.

Diante das diferenças significativas entre os grupos estudados, a utilização do THAAS se mostra de extrema importância na rotina clínica, sugerindo que o mesmo possa ser incluído na bateria da avaliação fonoaudiológica, como também nas avaliações de acompanhamento de deficientes auditivos. Além de que, sua adição ao trabalho de reabilitação auditiva, permitirá monitorar a evolução terapêutica da criança neste âmbito.

\section{Conclusão}

O desempenho das crianças com perda auditiva condutiva e sensório-neural de grau leve no THAAS foi inferior àquelas do grupo controle, sendo o pior comprometimento para as perdas sensório-neurais. A idade e o gênero das crianças não interferiram nos erros de desatenção, impulsividade e em suas habilidades para sustentar a atenção, nos três grupos estudados.

5. Asbjørnsen A, Holmefjord A, Reisaeter S, Møller P, Klausen $\mathrm{O}$, Prytz B, et al. Lasting auditory attention impairment after persistent middle ear infections: a dichotic listening study. Dev Med Child Neurol. 2000;42(7):481-6.

6. Klausen O, Moller P, Holmefjord A, Reisaeter S. Lasting effects on otites media with effusion on language skill ans listening performance. Acta Otolaryngol. 2000;543:73-6.

7. Roberts J, Hunter L, Gravel J, Rosenfeld R, Berman S, Haggard $\mathrm{M}$, et al. Otitis media, hearing loss, and language learning: controversies and current research. J Dev Behav Pediatr. 2004;25(2):110-22. 
8. Paradise JL, Dollaghan CA, Campbell TF, Feldman HM, Bernard BS, Colborn DK, et al. Otitis media and tympanostomy tube insertion during the first three years of life: developmental outcomes at the age of four years. Pediatrics. 2003;112:265-77.

9. Roush J. Screening for hearing loss and otitis media: basic principles. In: Roush J, editor. Screening for hearing loss and otitis media in children. San Diego, California: Singular; 2001. p.3-32.

10. Bess FH, Dodd-Murphy J, Parker RA. Children with minimal sensorineural hearing loss: prevalence, educational performance, and functional status. Ear Hear. 1998;19:339-54.

11. Wake M, Hughes E, Poulakis Z, Collins C, Rickards FW. Outcomes of mild-profound hearing impairment at age 7-8 years: a population study. Ear Hear. 2004;25:1-8.

12. Wake M, Tobin S, Cone-Wesson B, Dahl HH, Gillam L, McCormick L. Slight/Mild Sensorineural Hearing Loss in Children. Pediatrics. 2006;118:1842-51.

13. Gomes H, Molholm S, Christodoulou C, Ritter W, Cowan $\mathrm{N}$. The development of auditory attention in children. Front Biosci. 2000;1(5):D108-20.

14. Ward A. Attention-A Neuropsychological Perspective. Psychology Press. Hove-New York. p. 133. 2004.

15. Northern JL, Downs M. Hearing in children. 4th ed. Baltimore: Willians Wilkins; 1991.

16. Feniman MR, Ortelan RR, Lauris JRP, Campos CF, Cruz MS. Proposta de instrumento comportamental para avaliar a atenção auditiva sustentada. Rev Bras Otorrinolaringol 2007;73(4):523-7.

17. Neter J, Kutner MH, Wasserman W, Nachtsheim CJ. Applied Linear Statistical Models. 4ed. McGraw-Hill/Irwin: New York; 1996.

18. Greenberg LM, Waldman ID. Developmental normative data on the test of variables of attention (T.O.V.A.). J Chil Psychol Psychiatry 1993;34:1019-30.

19. Someya T, Sakado K, Seki T, Kojima M, Reist C, Tang SW, et al. The Japanese version of the Barratt Impulsiveness Scale, 11th version (BIS-11): its reliability and validity. Psychiatry Clin Neurosci. 2001; 55:111-4.
20. Feniman MR. Aplicação do teste de atenção auditiva FC2 em crianças normais [tese]. São Paulo: Faculdade de Odontologia de Bauru, Universidade de São Paulo; 2004.

21. Groot AS, Sonneville LMJ, Stins JF, Boomsma DI. Familial influences on sustained attention and inhibition in preschoolers. J Chil Psychol Psychiatry. 2004; 45:306-14.

22. Tinius TP. The intermediate visual and auditory continuous performance tests as a neuropsychological measure. Arch Clin Neuropsychol. 2003; 18(2):199-214.

23. Andalibi A, Li JD, Webster P, Lim DJ. Advances in treating middle ear infections in children. Hear Rev [on line] $2001 \mathrm{Sept}$ [consultado em 2008]. Disponível:http://www.hearing review. com/issues/articles/2001-09-02.asp.

24. Costa SMB, Costa Filho OA, Cardoso MRA. A percepção auditiva de crianças em idade escolar: associações entre os dados de anamnese e a avaliação audiológica - resultados preliminares. Pediatr Mod. 2001; 37:602-10.

25. Bess FH. School - aged children with minimal sensorineural hearing loss. Hear J. 1999; 52:10-6.

26. Paradise JL, Dollaghan CA, Campbell TF, Feldman HM, Bernard BS, Colborn DK et al. Language, speech sound production, and cognition in three-year-old children in relation to otitis media in their first three years of life. Pediatrics. 2000;105:1119-30.

27. Paradise JL, Feldman HM, Campbell TF, Dollaghan CA, Rockette HE, Pitcairn DL et al. Tympanostomy tubes and developmental outcomes at 9 to 11 years of age. N Eng J Med. 2007;248-61.

28. Keith RW. ACPT: Auditory Continuous Performance Test. San Antonio: Psychological Corporation; 1994.

29. Riccio CA, Cohen MJ, Hynd GW, Keith RW. Validity of the Auditory Continuous Test in differentiating central processing auditory disorders with and withot ADHD. J Learn Disabil. 1996;29:561-6.

30. Neijenhuis K, Tschur H, Snik A. The effect of mild hearing impairment on auditory processing tests. J Am Acad Audiol. 2004;15:6-16. 